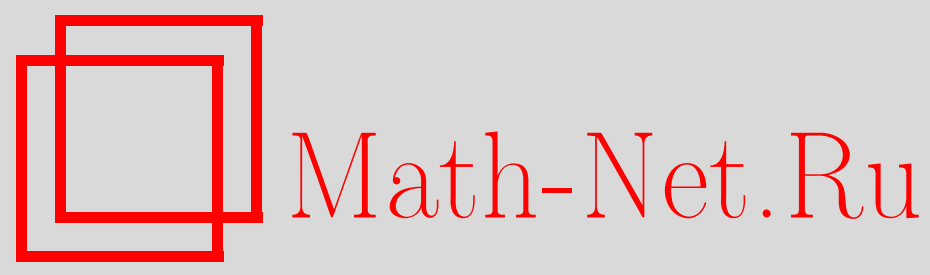

Р. Р. Гадыльшин, О рассеянии на цилиндрической ловушке в критическом случае, Матем. заметки, 2003, том 73, выпуск 3, 355-370

DOI: https://doi.org/10.4213/mzm194

Использование Общероссийского математического портала Math-Net.Ru подразумевает, что вы прочитали и согласны с пользовательским соглашением http://www . mathnet.ru/rus/agreement

Параметры загрузки:

IP : 54.224 .60 .19

26 апреля 2023 г., 11:38:00

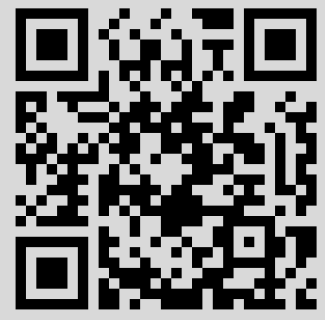




\section{О РАССЕЯНИИ НА ЦИЛИНДРИЧЕСКОЙ ЛОВУШКЕ В КРИТИЧЕСКОМ СЛУЧАЕ}

\section{P.P. Гадыльшин}

Рассмотрен двумерный аналог резонатора Гельмгольца со стенками конечной толщины в критическом случае, когда существует частота такая, что она является одновременно пределом полюсов как порождаемых ограниченной компонентой резонатора, так и узкого канала связи. В предположении, что предельная частота является простой для ограниченной компоненты, методом согласования асимптотических разложений построены асимптотики двух полюсов, сходящихся к этой частоте. Получены явные формулы для главных членов асимптотик полюсов и решения задачи рассеяния.

Библиографияя: 17 названий.

В работе рассматривается случай, когда сечение цилиндрического рассеивателя в пределе гомеоморфно кольцу, а возмушенная область получается вырезанием узкого канала $\varkappa_{\varepsilon}$, соединяющего обе несвязные компоненты внешности предельного сечения (ограниченную $\Omega^{\text {in }}$ и неограниченную $\Omega^{\text {ex }}$ ) и имеющего “диаметр” порядка $\varepsilon \ll 1$. Соответствующая математическая модель (как для возмущенной, так и для предельных задач) описьвается краевой задачей Неймана для уравнения Гельмгольца. Известно [1], что аналитическое продолжение решения возмущенной задачи в этом случае, в отличие от случая граничньх условий Дирихле [2], имеет две серии полюсов с мальми мнимыми частями. Предельньм множеством для первой серии является множество $\Sigma^{\text {in }}$ собственных частот (корней из собственных значений) задачи Неймана для $-\Delta$ (предельной внутренней задачи). Для второй серии предельное множество - это множество $\Sigma^{\mathrm{ch}}=\{m \pi / h\}_{m=1}^{\infty}$, где $h$ - длина канала связи. В [3] показано, что в случаях, когда предельная частота $k_{0}$ является простой собственной частотой из $\Sigma^{\text {in }}$, отделенной от $\Sigma^{\mathrm{ch}}$, либо, наоборот, когда $k_{0} \in \Sigma^{\mathrm{ch}} \backslash \Sigma^{\text {in }}$, у аналитического продолжения функции Грина возмущенной задачи существует по единственному полюсу первого порядка, сходящемуся при $\varepsilon \rightarrow 0$ к $k_{0}$, и каждому из них соответствует по одной обобщенной собственной функции. В [4] методом согласования асимптотических разложений [5]-[7] были получены явные формулы для главньх членов асимптотик как этих характеристик, так и пиков решений задачи рассеяния при вешественных частотах, близких к таким $k_{0}$. Из этих формул следовало, что в обоих случаях во внешности резонатора (т.е. вне ограниченной компоненты и вне канала связи) решение возмущенной задачи отличается от решения предельной задачи в неограниченной компоненте на величину $O(1)$. Поведение

Работа выполнена при поддержке Российского фонда фундаментальных исследований, гранты № 02-01-00768 и № 00-15-96038, и Министерства образования РФ, грант Е00-1.0-53. 
же решения возмушенной задачи в ограниченной компоненте (в ловушке) в этих случаях различалось существенно: при частотах, близких к $\Sigma^{\mathrm{in}} \backslash \Sigma^{\mathrm{ch}}$, оно имело порядок $\varepsilon^{-1}$, а при частотах, близких к $\Sigma^{\mathrm{ch}} \backslash \Sigma^{\mathrm{in}}$, было ограниченньп. В настоящей работе анализируется критическая ситуация, когда предельная частота $k_{0}$ одновременно является простой собственной частотой предельной внутренней задачи и принадлежит множеству $\Sigma^{\text {ch }}$. Результаты работы анонсированы в [8].

1. Постановка задачи, предварительные сведения и формулировка результатов. Пусть $\Omega^{\text {in }}$ и $\Omega$ - односвязные ограниченные области в $\mathbb{R}^{2}, \bar{\Omega}{ }^{\text {in }} \subset \Omega, \Omega^{\mathrm{ex}}=$ $\mathbb{R}^{2} \backslash \bar{\Omega}, \partial \Omega^{\mathrm{in}(\mathrm{ex})} \in C^{\infty}, x=\left(x_{1}, x_{2}\right)$. Предполагается, что $\Omega^{\text {in }}$ в окрестности начала координат совпадает с полуплоскостью $x_{2}>0$, область $\Omega^{\mathrm{ex}}$ в окрестности точки $x^{(0)}=(0,-h), h>0,-$ с полуплоскостью $x_{2}<-h$, а интервал $[0,-h]$ на оси $O x_{2}$ не содержит точек из $\Omega^{\text {in }} \cup \Omega^{\text {ex }}$. Области $\Omega^{\text {in }}$ и $\Omega^{\text {ex }}$ являются соответственно внутренностью и внешностью резонатора

$$
\Omega_{\varepsilon}=\Omega^{\mathrm{in}} \cup \Omega^{\mathrm{ex}} \cup \varkappa_{\varepsilon}
$$

где $\varkappa_{\varepsilon}=\left(\varepsilon \omega_{-}, \varepsilon \omega_{+}\right) \times[0,-h]-$ канал связи, $\omega_{-}<\omega_{+}-$любые числа.

Известно, что рассеяние как $H$-поляризованных полей на идеально проводящем цилиндре с сечением $\Omega_{\varepsilon}$, так и плоских акустических полей на идеально жестком цилиндре с сечением $\Omega_{\varepsilon}$, определяется решением краевой задачи

$$
\begin{aligned}
& \left(\Delta+k^{2}\right) u_{\varepsilon}=F, \quad x \in \Omega_{\varepsilon}, \quad \frac{\partial u_{\varepsilon}}{\partial \nu}=0, \quad x \in \partial \Omega_{\varepsilon} \\
& u_{\varepsilon}=O\left(\frac{1}{r^{1 / 2}}\right), \quad \frac{\partial u_{\varepsilon}}{\partial r}-i k u_{\varepsilon}=o\left(\frac{1}{r^{1 / 2}}\right), \quad r \rightarrow \infty
\end{aligned}
$$

где $r=|x|, \nu$ - внешняя нормаль, $k>0$, а $F$ - квадратично интегрируемая функция с ограниченньм носителем в $\Omega^{\mathrm{ex}}$. В случае акустического рассеяния $u_{\varepsilon}-$ потенциал скорости. Для $H$-поляризации $F$ - третья компонента вектора $-\operatorname{rot} \mathbf{j}$ в случае, когда вектор тока $\mathbf{j}$ перпендикулярен образующей, а электромагнитное поле имеет вид $\mathbf{H}_{\varepsilon}=$ $\left\{0,0, u_{\varepsilon}\right\}, \mathbf{E}_{\varepsilon}=-i k^{-1}\left(\operatorname{rot} \mathbf{H}_{\varepsilon}-\mathbf{j}\right)$. Всюду далее под предельной внутренней (внешней) задачей будем понимать краевую задачу Неймана для уравнений Гельмгольца в $\Omega^{\text {in }}$ (в $\left.\Omega^{\mathrm{ex}}\right)$.

Известно (см., например, [9]), что для положительных $k$ возмушенная краевая задача (1.1), (1.2) и предельная внешняя задача однозначно разрешимы и их функции Грина допускают аналитическое продолжение в комплексную плоскость с разрезом вдоль отрицательной вещественной полуоси, которое имеет дискретное (при фиксированном $\varepsilon$ ) множество полюсов $\Sigma_{\varepsilon}$ и $\Sigma^{\mathrm{ex}}$ соответственно, лежащее ниже вещественной оси. Как уже отмечалось выше, в [1] было доказано, что в любой малой окрестности ненулевого элемента $k_{0} \in \Sigma=\Sigma^{\text {in }} \cup \Sigma^{\text {ex }} \cup \Sigma^{\text {ch }}$ при всех достаточно малых $\varepsilon$ лежит полюс $\tau_{\varepsilon} \in \Sigma_{\varepsilon}$, и, наоборот, если компакт $K$ отделен от $\Sigma$, то $K \cap \Sigma_{\varepsilon}=\varnothing$ при любых достаточно малых $\varepsilon$.

В случаях, когда $k_{0} \in \Sigma_{1}^{\text {in }} \backslash \Sigma^{\mathrm{ch}}$, где $\Sigma_{1}^{\text {in }}$-множество ненулевых, простых собственных частот предельной внутренней задачи, и когда $k_{0} \in \Sigma^{\text {ch }} \backslash \Sigma^{\text {in }}$, существует по одному полюсу, сходящемуся к $k_{0}$ (см. [4]). Так как решения (1.1), (1.2) рассматриваются при $k>0$, а полюс $\tau_{\varepsilon}$ комплексньй, то в обоих случаях решения испытьвают наибольшие возмущения, когда

$$
k=k(\varepsilon)=\operatorname{Re} \tau_{\varepsilon}+O\left(\operatorname{Im} \tau_{\varepsilon}\right)
$$


Такие положительные частоты будем назьвать пиковыми. Обозначим через $S(t)$ и $S^{\text {ех }}(t)$ круги радиуса $t$ с центром в начале координат и в точке $x^{(0)}$ соответственно, через $G^{\text {in }(\mathrm{ex})}(x, y, k)$ функцию Грина предельной внутренней (внешней) задачи, через $u^{\mathrm{ex}}(x ; k)$ решение предельной внешней задачи, а через $\psi$ нормированную в $L_{2}\left(\Omega^{\text {in }}\right)$ собственную функцию, соответствующую простой частоте $k_{0} \in \Sigma_{1}^{\text {in }}$. В [4] было показано, что при пиковых частотах главные члены асимптотик возмущенной задачи $(1.1),(1.2)$ имеют вид

$$
\begin{aligned}
& u_{\varepsilon}(x ; k(\varepsilon)) \sim \frac{1}{\varepsilon} A_{1} \psi(x), \quad x \in \Omega^{\mathrm{in}} \backslash S\left(\varepsilon^{1 / 2}\right), \\
& u_{\varepsilon}(x ; k(\varepsilon)) \sim A_{2} G^{\mathrm{ex}}\left(x, x^{(0)}, k_{0}\right)+u^{\mathrm{ex}}\left(x ; k_{0}\right), \quad x \in\left(\Omega^{\mathrm{ex}} \backslash S^{\mathrm{ex}}\left(\varepsilon^{1 / 2}\right)\right) \cap S(R),
\end{aligned}
$$

для $k(\varepsilon) \rightarrow k_{0} \in \Sigma_{1}^{\text {in }} \backslash \Sigma^{\text {ch }}$ и

$$
\begin{aligned}
& u_{\varepsilon}(x ; k(\varepsilon)) \sim B_{1} G^{\text {in }}\left(x, 0, k_{0}\right), \quad x \in \Omega^{\mathrm{in}} \backslash S\left(\varepsilon^{1 / 2}\right), \\
& u_{\varepsilon}(x ; k(\varepsilon)) \sim B_{2} G^{\mathrm{ex}}\left(x, x^{(0)}, k_{0}\right)+u^{\mathrm{ex}}\left(x, k_{0}\right), \quad x \in\left(\Omega^{\mathrm{ex}} \backslash S^{\mathrm{ex}}\left(\varepsilon^{1 / 2}\right)\right) \cap S(R),
\end{aligned}
$$

для $k(\varepsilon) \rightarrow k_{0} \in \Sigma^{\text {ch }} \backslash \Sigma^{\text {in }}$, где $A_{j}$ и $B_{j}$ - некоторые явно вычисляемые константы, а $R>0$ - любое число.

Из (1.4) и (1.6) следует, что при пиковых частотах в $\Omega^{\text {ех }}$ в обоих случаях решения возмущенной задачи отличаются от решения предельной внешней задачи на $O(1)$. Именно это отличие было замечено Рэлеем [10] для классического резонатора (которьй представляет собой сферу с мальм отверстием связи). Будем назьвать этот эффект внешним резонансом. С другой стороны, из (1.3) и (1.5) следует, что при пиковых частотах в $\Omega^{\text {in }}$ решения возмущенной задачи в этих случаях отличаются существенно друг от друга: для $k(\varepsilon) \rightarrow k_{0} \in \Sigma^{\text {ch }} \backslash \Sigma^{\text {in }}$ решение ограничено, а для $k(\varepsilon) \rightarrow k_{0} \in \Sigma_{1}^{\text {in }} \backslash \Sigma^{\text {ch }}$ оно растет как $O\left(\varepsilon^{-1}\right)$. Будем называть ситуацию (1.3) внутренним резонансом. Заметим, что для трехмерного резонатора со стенками конечной толщины резонанс в [11] понимается именно в этом смысле, а асимптотики решений при $k(\varepsilon) \rightarrow k_{0} \in \Sigma^{\text {ch }} \backslash \Sigma^{\text {in }}$ и $k(\varepsilon) \rightarrow k_{0} \in \Sigma_{1}^{\text {in }} \backslash \Sigma^{\text {ch }}$ построены в [12], [13]. Так как (1.3) и (1.5) разнятся довольно сильно, возникает естественньй вопрос о поведении решения задачи рассеяния при частотах, близких к $k_{0} \in \Sigma_{1}^{\text {in }} \cap \Sigma^{\text {ch }}$. Заметим, что предлагаемьй для анализа критический случай не такой уж и вырожденный, так как для фиксированной “основной” ловушки $\Omega^{\text {in }}$ такая ситуация легко достигается соответствующим изменением длины канала связи (см. определение $\left.\Sigma^{\mathrm{ch}}\right)$.

Всюду далее используются следующие обозначения: $\omega$-интервал $\left(\omega_{-}, \omega_{+}\right),|\omega|=$ $\omega_{+}-\omega_{-}, x^{*}=\left(x_{1},-x_{2}\right)$,

$$
\sigma=\lim _{R \rightarrow \infty} \int_{\partial S(R)}\left|G^{\mathrm{ex}}\left(x, x^{(0)}, k_{0}\right)\right|^{2} d s
$$

Основной целью работы является доказательство следующего утверждения. 
Teоpema 1.1. Пусть $k_{0}=m \pi / h \in \Sigma_{1}^{\mathrm{in}} \cap \Sigma^{\mathrm{ch}}$. Тогдa

а) существует два полюса $\tau_{\varepsilon}^{(n)} \in \Sigma_{\varepsilon}, n=1,2$, сходящ,ихся $к k_{0}$ и имеющ, их асимптотики

$$
\tau_{\varepsilon}^{(n)}=k_{0}+\sum_{i=1}^{\infty} \sum_{j=0}^{i-1} \varepsilon^{i / 2} \ln ^{j} \varepsilon \tau_{i, j}^{(n)},
$$

$\tau_{1,0}^{(n)}=(-1)^{n} \psi(0)\left(\frac{|\omega|}{2 h}\right)^{1 / 2}, \quad \tau_{2,1}^{(n)}=(-1)^{n} \frac{4 k_{0}}{\pi \psi(0)}\left(\frac{|\omega|}{2 h}\right)^{1 / 2}, \quad \operatorname{Im} \tau_{2,0}^{(n)}=-\frac{1}{2} \frac{|\omega|}{h} k_{0}^{2} \sigma ;$

б) $п р и k$, близких $к k_{0}$, для решения краевой задачи (1.1), (1.2) и его аналитического продолэсения справедливо представление

$$
u_{\varepsilon}(x ; k)=-\sum_{n=1}^{2} \frac{\Psi_{\varepsilon}^{(n)}(x)}{\left(\tau_{\varepsilon}^{(n)}\right)^{2}-k^{2}} \int_{\mathbb{R}^{2}} \Psi_{\varepsilon}^{(n)}(y) F(y) d y+\widetilde{u}_{\varepsilon}(x ; k),
$$

әде $\widetilde{u}_{\varepsilon}-$ голоморфная функиия по переменной $k$. Eсли $\operatorname{supp} F \subset \Omega^{\mathrm{ex}}$, то $\widetilde{u}_{\varepsilon}$ сходится в $\Omega^{\mathrm{ex}}$ к решению предельной внешней задачи в $L_{2, \mathrm{loc}}\left(\Omega^{\mathrm{ex}}\right)($ m.е. на любом компакте из $\left.\Omega^{\mathrm{ex}}\right)$ и к нулю в $\Omega^{\text {in }} \cup \varkappa_{\varepsilon}$ по норме $L_{2}$;

в) для $\Psi_{\varepsilon}^{(n)}$ справедливы следующие асимптотики в $L_{2, \mathrm{loc}}\left(\mathbb{R}^{2}\right)$ :

$$
\begin{aligned}
& \Psi_{\varepsilon}^{(n)}(x)=\frac{(-1)^{n}}{\sqrt{2}} \psi(x)+o(1), \quad x \in \Omega^{\text {in }} \backslash S\left(\varepsilon^{1 / 2}\right), \\
& \Psi_{\varepsilon}^{(n)}(x)=\frac{(-1)^{n}}{\sqrt{2}} \psi(0)(1+o(1)), \quad x \in S\left(2 \varepsilon^{1 / 2}\right), \\
& \Psi_{\varepsilon}^{(n)}(x)=\frac{1}{\varepsilon^{1 / 2}}\left(\frac{1}{h|\omega|}\right)^{1 / 2}\left(\sin \left(k_{0} x_{2}\right)+o(1)\right), \quad x \in \varkappa_{\varepsilon} \backslash\left(S^{\mathrm{ex}}\left(\varepsilon^{1 / 2}\right) \cup S\left(\varepsilon^{1 / 2}\right)\right), \\
& \Psi_{\varepsilon}^{(n)}(x)=\varepsilon^{1 / 2} \frac{(-1)^{m+1} k_{0}}{\pi}\left(\frac{|\omega|}{h}\right)^{1 / 2}\left(\ln \varepsilon+\frac{\pi}{|\omega|} X\left(\frac{\left(x-x^{(0)}\right)^{*}}{\varepsilon}\right)+o(1)\right), \\
& \left.\Psi_{\varepsilon}^{(n)}(x)=\varepsilon^{1 / 2}\left((-1)^{m} k_{0}\left(\frac{|\omega|}{h}\right)^{1 / 2} G^{\operatorname{ex}}\left(2 \varepsilon^{1 / 2}\right), x^{(0)}, k_{0}\right)+o(1)\right), \quad x \in \Omega^{\mathrm{ex}} \backslash S^{\operatorname{ex}}\left(\varepsilon^{1 / 2}\right),
\end{aligned}
$$

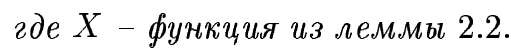

Из (1.7) и (1.8) вытекает, что в рассматриваемом случае имеются два "равноправных" пика решения задачи (1.1), (1.2), наблюдаемые при пиковых частотах

$$
k=k(\varepsilon)=k_{0}+\varepsilon^{1 / 2} \tau_{1,0}^{(n)}+\varepsilon \ln \varepsilon \tau_{2,1}^{(n)}+\varepsilon^{2}(t+o(1)),
$$

где $t$ - любое действительное число. Подставляя (1.10) и асимптотики из пункта в) теоремы в (1.9), получаем, что решения задачи рассеяния при таких $k$ имеют асимптотики

$$
u_{\varepsilon}(x ; k) \sim \frac{1}{\varepsilon^{1 / 2}} c_{F}^{(n)}(t) \psi(x), \quad x \in \Omega^{\text {in }} \backslash S\left(\varepsilon^{1 / 2}\right),
$$




$$
\begin{gathered}
u_{\varepsilon}(x ; k) \sim \frac{1}{\varepsilon^{1 / 2}} c_{F}^{(n)}(t) \psi(0), \quad x \in S\left(2 \varepsilon^{1 / 2}\right), \\
u_{\varepsilon}(x ; k) \sim \frac{1}{\varepsilon}(-1)^{n} c_{F}^{(n)}(t)\left(\frac{2}{h|\omega|}\right)^{1 / 2} \sin \left(k_{0} x_{2}\right), \quad x \in \varkappa_{\varepsilon} \backslash\left(S^{\mathrm{ex}}\left(\varepsilon^{1 / 2}\right) \cup S\left(\varepsilon^{1 / 2}\right)\right), \\
u_{\varepsilon}(x ; k) \sim c_{F}^{(n)}(t)(-1)^{m+n+1} \frac{k_{0}}{\pi}\left(\frac{2|\omega|}{h}\right)^{1 / 2}\left(\ln \varepsilon+\frac{\pi}{|\omega|} X\left(\frac{\left(x-x^{(0)}\right)^{*}}{\varepsilon}\right)\right), \\
u_{\varepsilon}(x ; k) \sim c_{F}^{(n)}(t)(-1)^{m+n} k_{0}\left(\frac{2|\omega|}{h}\right)^{1 / 2} G^{\mathrm{ex}}\left(x, x^{(0)}, k\right)+u^{\mathrm{ex}}(x ; k), \\
x \in \Omega^{\mathrm{ex}} \backslash S^{\mathrm{ex}}\left(\varepsilon^{1 / 2}\right), \\
c_{F}^{(n)}(t)=\frac{(-1)^{m+n+1}}{2\left(t-\tau_{2,0}^{(n)}\right)}\left(\frac{|\omega|}{2 h}\right)^{1 / 2} u^{\mathrm{ex}}\left(x^{(0)} ; k_{0}\right),
\end{gathered}
$$

из которых следует, что при обеих пиковых частотах наблюдается внутренний резонанс в $\Omega^{\text {in }}$, но он отличается на порядок от (1.3).

2. Построение асимптотик. Обозначим

$$
\begin{aligned}
R_{t}^{\mathrm{in}(\mathrm{ex}), i, j}\left(D_{y}\right) & =\sum_{q=0}^{t} a_{i, j, q}^{\mathrm{in}(\mathrm{ex})} \frac{\partial^{q}}{\partial y_{1}^{q}} \\
\psi_{\varepsilon}^{\text {in }(\mathrm{ex})}(x, k) & =\sum_{i=0}^{\infty} \sum_{j=0}^{i} \varepsilon^{i / 2} \ln ^{j} \varepsilon\left(k_{0}^{2}-k^{2}\right) R_{[(i-j) / 2]}^{\mathrm{in}(\mathrm{ex}), i, j}\left(D_{y}\right) G^{\mathrm{in}(\mathrm{ex})}\left(x, x_{0}^{\mathrm{in}(\mathrm{ex})}, k\right),
\end{aligned}
$$

где $x_{0}^{\text {in }}$ - начало координат, $x_{0}^{\mathrm{ex}}=x^{(0)}, a_{i, j, q}^{\text {in }(\mathrm{ex})}-$ постоянные, $[N]-$ целая часть $N$. Коэффициенты $\psi_{\varepsilon}^{\text {in }(\mathrm{ex})}(x, k)$ аналитичны по переменной $k$ в некоторой комплексной окрестности точки $k_{0}$, являются решением уравнения $\left(\Delta+k^{2}\right) U=0$ в $\Omega^{\text {in(ex) }}$ и $\partial U / \partial \nu=0$ на $\partial \Omega^{\text {in }(\text { ех })} \backslash\left\{x_{0}^{\text {in(ex) }}\right\}$, а при положительных $k$ коэффициенты ряда $\psi_{\varepsilon}^{\text {ex }}(x, k)$ удовлетворяют и условию излучения (1.2). Поэтому вне канала связи и малых окрестностей его концов полные асимптотики обобщенных собственных функций $\Psi_{\varepsilon}^{(n)}$ будем искать, следуя [4], в виде

$$
\psi_{\varepsilon}^{(n)}(x)=\psi_{\varepsilon}^{\mathrm{in}(\mathrm{ex})}\left(x, \tau_{\varepsilon}^{(n)}\right), \quad x \in \Omega^{\mathrm{in}(\mathrm{ex})} \backslash S^{\mathrm{in}(\mathrm{ex})}\left(\varepsilon^{1 / 2}\right),
$$

где $S^{\text {in }}(t)=S(t)$, a $R_{t}^{\text {in }(\mathrm{ex}), i, j}$ в определении $\psi_{\varepsilon}^{\text {in(ex) }}$ зависят от $n$.

ЗАмечаниЕ 2.1. Так как строгой нормировки на $\Psi_{\varepsilon}^{(n)}$ в $(1.9)$ не накладывается, то под $\psi_{\varepsilon}^{(n)}$ в (2.1) понимается функция, равная $\Psi_{\varepsilon}^{(n)}$ с точностью до скалярного множителя $\alpha_{n}(\varepsilon)$. В п. 3 будет показано, что $\alpha_{n}(\varepsilon)=1+o(1)$ при $\varepsilon \rightarrow 0$.

ЗАМЕЧАНИЕ 2.2. Построение асимптотик для обоих полюсов и соответствующих обобщенных собственных функций одинаково. По этой причине, а также, чтобы не перегружать обозначения в тексте индексом соответствия конкретному полюсу ( “ $n$ "), этот индекс в обозначениях по возможности будет опускаться (в том числе и во введенных выше обозначениях). 
В малой окрестности канала связи асимптотики $\psi_{\varepsilon}^{(n)}$ строятся в виде

$$
\begin{aligned}
& \psi_{\varepsilon}(x)=\sum_{i=-1}^{\infty} \sum_{j=0}^{i} \varepsilon^{i / 2} \ln ^{j} \varepsilon w_{i, j}\left(x_{2}\right), \quad x \in \varkappa_{\varepsilon} \backslash\left(S^{\mathrm{ex}}\left(\varepsilon^{1 / 2}\right) \cup S^{\mathrm{in}}\left(\varepsilon^{1 / 2}\right)\right), \\
& \psi_{\varepsilon}(x)=\sum_{i=1-\beta_{\mathrm{in}(\mathrm{ex})}}^{\infty} \sum_{j=0}^{i} \varepsilon^{i / 2} \ln ^{j} \varepsilon v_{i, j}^{\mathrm{in}(\mathrm{ex})}\left(\frac{x^{\mathrm{in}(\mathrm{ex})}}{\varepsilon}\right), \quad x \in S^{\mathrm{in}(\mathrm{ex})}\left(2 \varepsilon^{1 / 2}\right),
\end{aligned}
$$

где $x^{\text {in }}=x, x^{\mathrm{ex}}=\left(x-x^{(0)}\right)^{*}, \beta_{\text {in }}=1, \beta_{\mathrm{ex}}=0$. Поясним вид главных членов в $(2.1)$ и (2.2). При формальном переходе к пределу в $\psi_{\varepsilon}^{\text {in }}(x, k)$ при $\varepsilon \rightarrow 0$ и $k \rightarrow k_{0}$ получаем, что

$$
\psi_{\varepsilon}^{\text {in }}(x, k) \rightarrow R_{0}^{\text {in }, 0,0} \psi(0) \psi(x) .
$$

С другой стороны, в [4] показано, что в случае $k_{0} \in \Sigma_{1}^{\text {in }} \backslash \Sigma^{\mathrm{ch}}$ обобщенная собственная функция сходится к собственной функции $\psi$ (продолженной нулем вне $\Omega^{\text {in }}$ ), а в случае $k_{0} \in \Sigma^{\mathrm{ch}} \backslash \Sigma^{\text {in }}$ она в главном представима функцией

$$
\frac{1}{\varepsilon^{1 / 2}}\left(\frac{2}{|\omega| h}\right)^{1 / 2} \sin \left(k_{0} t\right)
$$

в $\varkappa_{\varepsilon}$ и нулем вне $\varkappa_{\varepsilon}$. Поэтому при $k_{0} \in \Sigma_{1}^{\text {in }} \cap \Sigma^{\mathrm{ch}}$ естественно ожидать, что главньй член асимптотики обобщенной собственной функции будет представляться "линейной" комбинацией $\psi$ и (2.5). Последнее и приводит к указанным в (2.1) и (2.2) главным членам асимптотик и, в частности, к равенству

$$
w_{-1,0}(t)=b_{-1,0} \sin \left(k_{0} t\right) .
$$

Более того, так как $\psi$ - нормированная функция, а норма $(2.5)$ в $L_{2}\left(\varkappa_{\varepsilon}\right)$ также равна единище, то из соотношений (2.4) и (2.6) и предположения (в дальнейшем оправданного) сохранения нормировки главного члена асимптотики обобщенной функции для рассматриваемого в работе случая получаем равенство

$$
\left(R_{0}^{\text {in }, 0,0} \psi(0)\right)^{2}+\frac{b_{-1,0}^{2}|\omega| h}{2}=1 .
$$

Краевые задачи для коэффициентов рядов (2.3) получаются стандартной подстановкой (см. [4], [7]) рядов (1.7), (2.3) в (1.1) при $F=0$ и переходом к “внутренней" перемен-

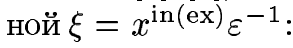

$$
\Delta v_{i, j}=-k_{0}^{2} v_{i-2, j}-\sum_{q=1}^{i-4} \sum_{t=0}^{q-1} \lambda_{q, t} v_{i-q-4, j-t}, \quad \xi \in \gamma_{\omega}, \quad \frac{\partial v_{i, j}}{\partial \nu}=0, \quad \xi \in \partial \gamma_{\omega}
$$

где $\lambda_{q, t}-$ коэффициенты ряда $\lambda_{\varepsilon}=\tau_{\varepsilon}^{2}-k_{0}^{2}$ при $\varepsilon^{q / 2} \ln ^{t} \varepsilon$,

$$
\gamma_{\omega}=(\omega \times(-\infty, 0]) \cup\left\{\xi: \xi_{2}>0\right\},
$$

а верхние индексы у $v_{q, t}^{\text {in (ex) }}$ опущены. 
Так как коэффициенты ряда (2.2) зависят только от $x_{2}$ (но понимаются как функции, определенные на $\left.\varkappa_{\varepsilon}\right)$, подставляя ряды $(1.7)$ и $(2.2)$ в (1.1) при $F=0$, получаем обыкновенные дифференциальные уравнения на $w_{j, i}$ :

$$
w_{i, j}^{\prime \prime}\left(x_{2}\right)+k_{0}^{2} w_{i, j}\left(x_{2}\right)+\sum_{q=1}^{i+1} \sum_{t=0}^{q-1} \lambda_{q, t} w_{i-q, j-t}\left(x_{2}\right)=0, \quad-h<x_{2}<0,
$$

решениями которых являются функции

$w_{i, j}\left(x_{2}\right)=-\frac{1}{k_{0}} \sum_{q=1}^{i+1} \sum_{t=0}^{q-1} \lambda_{q, t} \int_{-h}^{x_{2}} \sin \left(k_{0}\left(x_{2}-t\right)\right) w_{i-q, j-t}(t) d t+b_{i, j} \cos \left(k_{0} x_{2}\right), \quad j \geqslant 0$,

где $b_{i, j}$ - произвольные постоянные. Всюду далее коэффициенты ряда (2.2) выбраны в соответствии с (2.9). Легко видеть, что при таком определении

$$
w_{0,0}(t)=\tau_{1,0} k_{0} b_{-1,0}\left(t \cos \left(k_{0} t\right)+h \cos \left(k_{0} t\right)-\frac{1}{k_{0}} \sin \left(k_{0} t\right)\right)+b_{0,0} \cos \left(k_{0} t\right)
$$

Пусть $\rho=|\xi|, P_{j}(\xi)$ - однородные полиномы степени $j$, a $T_{j}(\xi)$ - однородные функции степени $j$, представимые в виде $P_{j+2 q}(\xi) \rho^{-2 q}$ при каком-нибудь целом $q \geqslant 1$ и удовлетворяющие граничному условию $\partial T_{j}(\xi) / \partial \xi_{2}=0$ при $\xi_{2}=0, \xi \neq 0$. Обозначим через $\tilde{\mathscr{A}}_{j}$ множество рядов вида

$$
T(\xi)=\sum_{q=-\infty}^{j} T_{q}(\xi)+\ln \rho \sum_{n=0}^{j} P_{q}(\xi)
$$

Далее обозначим через $w_{\varepsilon}(x)$ ряд (2.2). На суммах $U(x, \varepsilon)$ вида $w_{\varepsilon}(x)$ и $\psi_{\varepsilon}^{\text {in }(\text { ex })}\left(x, \tau_{\varepsilon}\right)$, где $\tau_{\varepsilon}$ - произвольная функция, имеющая асимптотику (1.7), определим операторы "переразложения" $K_{q}^{\text {in(ex) }}$ следующим, стандартньм для метода согласования, образом [7]. Разложим коэффициенты $U(x, \varepsilon)$ в ряды при $\mid x^{\text {in(ex) }} \rightarrow 0$ и перейдем к переменным $\xi=x^{\text {in }(\mathrm{ex})} \varepsilon^{-1}$ (в случае $U(x, \varepsilon)=\psi_{\varepsilon}^{\text {in }(\mathrm{ex})}\left(x, \tau_{\varepsilon}\right)$ функция $\tau_{\varepsilon}$ заменяется на ее асимптотический ряд). В полученном таким образом двойном ряду возьмем сумму членов $\varepsilon^{j} \ln ^{i} \varepsilon \Phi(\xi)$ при $j \leqslant q$. Эту сумму и назовем $K_{q}^{\text {in }(\mathrm{ex})}(U(x, \varepsilon))$.

Обозначим

$$
\begin{aligned}
& g^{\mathrm{in}}(k)=\lim _{x \rightarrow 0}\left(G^{\mathrm{in}}(x, 0, k)+\frac{1}{\pi} \ln r+\frac{\psi^{2}(0)}{k^{2}-k_{0}^{2}}\right), \\
& g^{\mathrm{ex}}(k)=\lim _{x \rightarrow x^{(0)}}\left(G^{\mathrm{ex}}\left(x, x^{(0)}, k\right)+\frac{1}{\pi} \ln \left|x-x^{(0)}\right|\right),
\end{aligned}
$$

а через $(\rho, \theta)$ обозначим полярные координаты. Из определений $\psi_{\varepsilon}^{\text {in }(\mathrm{ex})}, w_{\varepsilon}, K_{q}^{\text {in(ex })}$, paвенств $(2.6),(2.10)$ и асимптотик функций Грина предельных задач и их производных (см., например, [14]) вытекает 
ЛЕмма 2.1. Пусть $\tau_{\varepsilon}$ - произвольная функиия, имеющая асимптотику (1.7). Тогда для любого иелого $N \geqslant 0$ справедливы равенства

$$
\begin{aligned}
K_{N / 2}^{\mathrm{in}(\mathrm{ex})}\left(\psi_{\varepsilon}^{\mathrm{in}(\mathrm{ex})}\left(x, \tau_{\varepsilon}\right)\right) & =\sum_{i=1-\beta_{\text {in }(\mathrm{ex})}}^{N} \sum_{j=0}^{i} \varepsilon^{i / 2} \ln ^{j} \varepsilon V_{i, j}^{\mathrm{in}(\mathrm{ex})}(\xi) \\
K_{N / 2}^{\mathrm{in}(\mathrm{ex})}\left(w_{\varepsilon}(x)\right) & =\sum_{i=0}^{N} \sum_{j=0}^{i} \varepsilon^{i / 2} \ln ^{j} \varepsilon W_{i, j}^{\mathrm{in}(\mathrm{ex})}\left(\xi_{2}\right)
\end{aligned}
$$

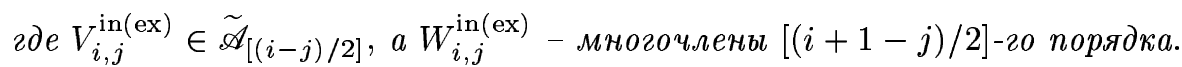

Справедливы представления

$$
\begin{aligned}
& V_{0,0}^{\text {in }}(\xi)=R_{0}^{\text {in }, 0,0} \psi^{2}(0), \\
& V_{1,0}^{\mathrm{in}(\mathrm{ex})}(\xi)=\beta_{\mathrm{in}(\mathrm{ex})} R_{0}^{\mathrm{in}(\mathrm{ex}), 1,0} \psi^{2}(0)+\frac{2}{\pi} k_{0} \tau_{1,0}\left(R_{0}^{\mathrm{in}(\mathrm{ex}), 0,0}\left(\ln \rho-\pi g^{\mathrm{in}(\mathrm{ex})}\left(k_{0}\right)\right)-\Pi_{0,0}\right), \\
& V_{k, k}^{\mathrm{in}(\mathrm{ex})}(\xi)=\frac{2 k_{0}}{\pi} \sum_{t=0}^{k-1} R_{0}^{\mathrm{in}(\mathrm{ex}), t, t} \tau_{k-t, k-t-1}, \quad k \geqslant 1, \\
& V_{k, j}^{\mathrm{in}(\mathrm{ex})}(\xi)=\widetilde{V}_{k, j}^{\mathrm{in}(\mathrm{ex})}(\xi)+\beta_{\mathrm{in}(\mathrm{ex})} a_{k, j, 0}^{\mathrm{in}(\mathrm{ex})} \psi^{2}(0) \\
& +\frac{2}{\pi} k_{0}\left(\tau_{1,0}\left(a_{k-1, j, 0}^{\mathrm{in}(\mathrm{ex})}\left(\ln \rho-\pi g^{\mathrm{in}(\mathrm{ex})}\left(k_{0}\right)\right)-\Pi_{k-1, j}\right)\right. \\
& \left.+\tau_{k, j}\left(R_{0}^{\text {in }(\mathrm{ex}), 0,0}\left(\ln \rho-\pi g^{\text {in }(\mathrm{ex})}\left(k_{0}\right)\right)-\Pi_{0,0}\right)\right), \quad k>1, \quad k>j, \\
& \Pi_{i, j}=\sum_{t=1}^{\infty} a_{2 t+i, j, t}^{\operatorname{in}(\mathrm{ex})}(t-1) ! \frac{\cos (t \theta)}{\rho^{t}}, \\
& \widetilde{V}_{2,0}^{\text {in }}(\xi)=R_{0}^{\text {in }, 0,0} \psi(0) \psi_{x_{1}}(0) \xi_{1}, \quad \tilde{V}_{2,0}^{\text {ex }}(\xi)=\widetilde{V}_{2,1}^{\text {in }(\mathrm{ex})}(\xi)=0,
\end{aligned}
$$

əде $\widetilde{V}_{i, j}^{\mathrm{in}(\mathrm{ex})}$ не зависит от $\tau_{q, s}, \Pi_{q-1, s}$ u $a_{q-1, s, 0}^{\mathrm{in}(\mathrm{ex})}$ npu $q \geqslant i u s \geqslant j$,

$$
\begin{gathered}
W_{i, i}^{\mathrm{in}}\left(\xi_{2}\right)=\tau_{i+1, i} b_{-1,0} h+b_{i, i}, \quad W_{i, i}^{\mathrm{ex}}\left(\xi_{2}\right)=(-1)^{m} b_{i, i}, \\
W_{k, j}^{\mathrm{in}}\left(\xi_{2}\right)=\widetilde{W}_{k, j}^{\mathrm{in}}\left(\xi_{2}\right)+b_{-1,0} h\left(\frac{1}{2} \tau_{k, j} \tau_{1,0}+\tau_{k+1, j}\right)+b_{k, j}, \\
W_{k, j}^{\mathrm{ex}}\left(\xi_{2}\right)=\widetilde{W}_{k, j}^{\mathrm{ex}}\left(\xi_{2}\right)+(-1)^{m} b_{k, j}, \quad k>j, \\
\widetilde{W}_{1,0}^{\mathrm{in}}\left(\xi_{2}\right)=b_{-1,0} k_{0} \xi_{2}, \quad \widetilde{W}_{1,0}^{\mathrm{ex}}\left(\xi_{2}\right)=(-1)^{m+1} b_{-1,0} k_{0} \xi_{2}, \\
\widetilde{W}_{k+1, k}^{\mathrm{in}}\left(\xi_{2}\right)=\alpha_{k}^{\mathrm{in}}, \quad \widetilde{W}_{k+1, k}^{\mathrm{ex}}\left(\xi_{2}\right)=\alpha_{k}^{\mathrm{ex}}, \quad k \geqslant 1, \quad \widetilde{W}_{2,0}^{\mathrm{in}(\mathrm{ex})}\left(\xi_{2}\right)=0,
\end{gathered}
$$

əде $\widetilde{W}_{i, j}^{\mathrm{in}(\mathrm{ex})}$ не зависят от $\tau_{q, s}$ u $b_{q, s}$ nри $q \geqslant i$ и $s \geqslant j, a \alpha_{k}^{\mathrm{in}(\mathrm{ex})}$ - некоторые постоянные.

Ряды $V_{i, j}^{\text {in(ex) }}\left(a\right.$, следовательно, и ряды $\left.\widetilde{V}_{i, j}^{\text {in(ex })} \in \mathscr{A}_{[(i-j) / 2]}\right)$ являются формальными асимптотическими решениями краевой задачи (2.8) при $\rho \rightarrow \infty, \xi_{2} \geqslant 0$, әде функиии $v_{q, t}$ заменены на $V_{q, t}^{\text {in(ex) }}$. 
Если $W_{0,0}^{\mathrm{ex}} \equiv 0$, то $W_{i, j}^{\mathrm{in}(\mathrm{ex})}\left(\right.$ a, следовательно, и $\left.\widetilde{W}_{i, j}^{\mathrm{in}(\mathrm{ex})}\right)$ являются формальньми асимптотическими решениями краевой задачи (2.8) при $\rho \rightarrow \infty, \xi_{2}<0$, әде функиии $v_{q, t}$ заменены на $W_{q, t}^{\text {in(ex) }}$.

Для согласования рядов (2.1)-(2.3) нужно показать существование решений краевых задач $(2.8)$, имеющих на бесконечности асимптотики, равные $V_{i, j}^{\text {in(ex) }}$ при $\xi_{2} \geqslant 0$ и равные $W_{i, j}^{\text {in(ex) }}$ при $\xi_{2}<0$. Пусть $0=\mu_{0}<\mu_{1} \leqslant \mu_{2} \leqslant \cdots-$ собственные частоты задачи Неймана для оператора $-d^{2} / d t^{2}$ на интервале $\omega, \beta_{q}(t)$ - соответствуюшие, нормированные в $L_{2}(\omega)$ собственные функции. Обозначим через $\widetilde{\mathscr{B}}_{q, n}$ множество рядов вида

$$
H(\xi)=R_{q}^{(0)}\left(\xi_{2}\right)+\sum_{j=1}^{\infty} R_{n}^{(j)}\left(\xi_{2}\right) \beta_{j}\left(\xi_{1}\right) \exp \left\{\mu_{j} \xi_{2}\right\}
$$

где $R_{i}^{(m)}(t)$ - многочлены порядка $i$. При отрищательных $n$ под $\widetilde{\mathscr{B}}_{q, n}$ понимается множество многочленов порядка $q$. Положим $\widetilde{\mathscr{B}}=\bigcup_{n, q} \widetilde{\mathscr{B}}_{q, n}$. Обозначим через $\mathscr{A}_{m}$ множество функций из $C^{\infty}\left(\gamma_{\omega}\right) \cap W_{2, \text { lос }}^{1}\left(\gamma_{\omega}\right)$, удовлетворяющих однородному условию Неймана на $\partial \gamma_{\omega}$ и имеющих на бесконечности дифференцируемые асимптотики из $\widetilde{\mathscr{A}}_{m}$ и $\widetilde{\mathscr{B}}$ при $\xi_{2} \geqslant 0$ и $\xi_{2}<0$ соответственно. Согласование асимптотических рядов проводится на основе леммы 2.1 и следующего утверждения, доказательство которого имеется в [4].

Лемма 2.2. Пусть $f \in \mathscr{A}_{N}$, яяд $V \in \widetilde{\mathscr{A}}_{N+2}$ является формальным асимптотическим решением уравнения $\Delta V=f$ при $\rho \rightarrow \infty, \xi_{2} \geqslant 0$, а многочлен $W\left(\xi_{2}\right)$ удовлетворяет уравнению $\Delta W=f+o(1)$ (или, что то же самое, уравнению $\left.W^{\prime \prime}=f+o(1)\right) n p u \rho \rightarrow \infty u \xi_{2}<0$.

Тогда существует функиия $v \in \mathscr{A}_{N+2}$, являющаяся решением краевой задачи

$$
\Delta v=f, \quad \xi \in \gamma_{\omega}, \quad \frac{\partial v}{\partial \nu}=0, \quad \xi \in \partial \gamma_{\omega}
$$

и имеющая при $\rho \rightarrow \infty$ дифферениируемые асимптотики

$$
\begin{array}{ll}
v(\xi)=V(\xi)+c_{0} \ln \rho+\sum_{j=1}^{\infty} c_{j} \frac{\cos (j \theta)}{\rho^{j}}, & \xi_{2} \geqslant 0, \\
v(\xi)=W\left(\xi_{2}\right)+q_{0}+O\left(\xi_{2}^{M} \exp \left\{\mu_{1} \xi_{2}\right\}\right), & \xi_{2}<0,
\end{array}
$$

әде $M \geqslant 0, q_{0}$ и $c_{i}$ - некоторые числа.

Существуют гармонические в $\gamma_{\omega}$ функиии $X \in \mathscr{A}_{0}$ и $Y \in \mathscr{A}_{1}$, имеющие на бесконечности асимптотики

$$
\begin{aligned}
& X(\xi)=\xi_{2}+q_{\omega}+O\left(\exp \left\{\mu_{1} \xi_{2}\right\}\right), \quad \xi_{2} \leqslant 0, \\
& X(\xi)=c_{\omega} \ln \rho+\sum_{j=1}^{\infty} c_{j}^{+} \frac{\cos (j \theta)}{\rho^{j}}, \quad \xi_{2} \geqslant 0, \\
& Y(\xi)=\xi_{1}+c^{\omega} \ln \rho+\sum_{j=1}^{\infty} b_{j} \frac{\cos (j \theta)}{\rho^{j}}, \quad \xi_{2} \geqslant 0, \\
& Y(\xi)=q^{\omega}+O\left(\exp \left\{\mu_{1} \xi_{2}\right\}\right), \quad \xi_{2}<0 .
\end{aligned}
$$


Из соображений четности следует, что при $\omega_{-}=-\omega_{+}$постоянные $q^{\omega}$ и $c^{\omega}$ равны нулю. Отсюда очевидным образом вытекает, что в общем случае

$$
c^{\omega}=0, \quad q^{\omega}=\frac{\omega_{+}+\omega_{-}}{2}
$$

Равенства $c_{\omega}=\pi^{-1}|\omega|, q_{\omega}=\pi^{-1}|\omega|\left(\ln \left(2|\omega| \pi^{-1}\right)-1\right)$ легко показать, используя конформное отображение полосы на $\gamma_{\omega}$ (см., например, [4], [15]).

Обозначим через $v_{\varepsilon}^{\text {in }(\mathrm{ex})}\left(x^{\mathrm{in}(\mathrm{ex})} / \varepsilon\right)$ ряды $(2.3)$, через $v_{\varepsilon, N}^{\text {in }(\mathrm{ex})}(\xi)$ частичные суммы этих рядов и перейдем к доказательству ключевого утверждения настоящей работы.

ТЕорема 2.1. Существуют функиия $\tau_{\varepsilon}$, имеющая асимптотику (1.7), и ря-

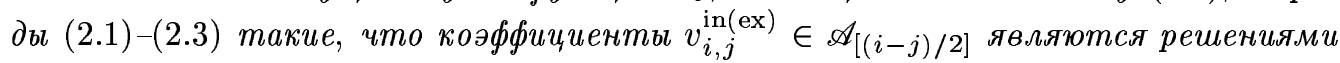
рекуррентной системы краевых задач (2.8), коэффициенты $w_{i, j}$ определяются равенствами (2.6), (2.9), для любого иелого $N \geqslant 0$ справедливы дифферениируемые асимптотические равенства

$$
\begin{gathered}
K_{N / 2}^{\mathrm{in}(\mathrm{ex})}\left(w_{\varepsilon}(x)\right)=v_{\varepsilon, N / 2}^{\mathrm{in}(\mathrm{ex})}(\xi)+O\left(\varepsilon^{N / 2} \xi_{2}^{M_{N}} \exp \left\{\mu_{1} \xi_{2}\right\}\right), \quad \xi_{2}<0, \\
K_{N / 2}^{\mathrm{in}(\mathrm{ex})}\left(\psi_{\varepsilon}^{\mathrm{in}(\mathrm{ex})}\left(x, \tau_{\varepsilon}\right)\right)=v_{\varepsilon, N / 2}^{\mathrm{in}(\mathrm{ex})}(\xi), \quad \xi_{2} \geqslant 0, \quad \rho \rightarrow \infty,
\end{gathered}
$$

а для коэффичиентов этих рядов справедливы следующ,ие соотношения:

$$
\begin{gathered}
b_{0,0}=0, \quad v_{0,0}^{\text {in }} \equiv R_{0}^{\text {in }, 0,0} \psi^{2}(0), \quad R_{0}^{\mathrm{ex}, 0,0}=(-1)^{m+1} R_{0}^{\text {in }, 0,0} \\
b_{-1,0}=\left(\frac{1}{h|\omega|}\right)^{1 / 2}, \quad R_{0}^{\text {in }, 0,0}= \pm \frac{1}{\psi(0)}\left(\frac{1}{2}\right)^{1 / 2}, \quad \tau_{1,0}= \pm \psi(0)\left(\frac{|\omega|}{2 h}\right)^{1 / 2}, \\
\tau_{2,0}=\frac{v_{1,1}^{\mathrm{ex}} \equiv \frac{2 k_{0} \tau_{1,0}}{\pi} R_{0}^{\mathrm{ex}, 0,0}, \quad \tau_{2,1}=\frac{4 k_{0}}{b_{-1,0} h \pi} R_{0}^{\mathrm{in}, 0,0}}{\tau_{1,0} b_{-1,0} h+R_{0}^{\text {in }, 0,0} \psi^{2}(0)} \\
\quad \times\left(b_{-1,0}\left(2 k_{0} q_{\omega}-\frac{1}{2} h \tau_{1,0}^{2}\right)-2 k_{0} \tau_{1,0} R_{0}^{\text {in }, 0,0}\left(g^{\text {in }}\left(k_{0}\right)+g^{\mathrm{ex}}\left(k_{0}\right)\right)\right) .
\end{gathered}
$$

ДокАЗАТЕЛЬСТво. Полагая $b_{0,0}=0$, в силу леммы 2.1 добиваемся равенств $(2.12)$, (2.13) для индекса “ех" при $N=0$. Аналогично, определяя $v_{0,0}^{\mathrm{ex}}$ в силу $(2.14)$ и накладывая дополнительное условие

$$
R_{0}^{\text {in }, 0,0} \psi^{2}(0)=\tau_{1,0} h b_{-1,0}
$$

где $R_{0}^{\text {in }, 0,0}, \tau_{1,0}$ и $b_{-1,0}$ - пока неизвестные постоянные (но подчиненные соотношению $(2.7))$, в силу леммы 2.1 (и определенного вьше значения $b_{0,0}$ ) добиваемся равенств $(2.12),(2.13)$ для индекса "in" при $N=0$. "Нулевой” шаг индукции завершен.

На следующем шаге, учитывая вид $\widetilde{W}_{1,0}^{\text {in,ex }}$ и $V_{1,0}^{\text {in,ex }}$, полагаем

$$
v_{1,0}^{\text {in }}=b_{-1,0} k_{0} X+A_{1,0}^{\text {in }}, \quad v_{1,0}^{\mathrm{ex}}=(-1)^{m+1} b_{-1,0} k_{0} X+A_{1,0}^{\mathrm{ex}}
$$


где $A_{1,0}^{\text {in }(\text { ex })}$ - пока также неопределенные постоянные. Легко видеть, что определенные таким образом функции являются решениями краевой задачи (2.8). Приравнивая асимптотики (степенные) функций $v_{1,0}^{\text {in }(\text { ех })}$ при $\rho \rightarrow \infty$ и $\xi_{2}<0$ к $W_{1.0}^{\text {in }(\text { ex })}$, в силу леммы 2.1 получаем уравнения

$$
\begin{gathered}
(-1)^{m+1} b_{-1,0} k_{0} q_{\omega}+A_{1,0}^{\mathrm{ex}}=(-1)^{m} b_{1,0}, \\
b_{-1,0} k_{0} q_{\omega}+A_{1,0}^{\mathrm{in}}=b_{-1,0} h\left(\frac{1}{2} \tau_{1,0}^{2}+\tau_{2,0}\right)+b_{1,0} .
\end{gathered}
$$

Аналогично, приравнивая асимптотики функций $v_{1,0}^{\text {in(ex) }}$ при $\rho \rightarrow \infty$ и $\xi_{2}>0$ к рядам $V_{1.0}^{\text {in(ex) }}$ до членов $O(1)$ включительно, получаем уравнения

$$
\begin{gathered}
b_{-1,0} k_{0} c_{\omega}=\frac{2}{\pi} k_{0} \tau_{1,0} R_{0}^{\mathrm{in}, 0,0} \\
(-1)^{m+1} b_{-1,0} k_{0} c_{\omega}=\frac{2}{\pi} k_{0} \tau_{1,0} R_{0}^{\mathrm{ex}, 0,0} \\
R_{0}^{\mathrm{in}, 1,0} \psi^{2}(0)-2 k_{0} \tau_{1,0} R_{0}^{\mathrm{in}, 0,0} g^{\mathrm{in}}\left(k_{0}\right)=A_{1,0}^{\mathrm{in}} \\
-2 k_{0} \tau_{1,0} R_{0}^{\mathrm{ex}, 0,0} g^{\mathrm{ex}}\left(k_{0}\right)=A_{1,0}^{\mathrm{ex}}
\end{gathered}
$$

где $R_{0}^{\text {in }, 1,0}$ - еще одна неизвестная постоянная. Из (2.17) и (2.18) получаем значение (2.14) для $R_{0}^{\mathrm{ex}, 0,0}, \mathrm{a}(2.20)$ определяет постоянную $A_{1,0}^{\mathrm{ex}}$. Разрешая систему уравнений $(2.7),(2.15),(2.17)$, получаем формулы $(2.14)$ для $R_{0}^{\text {in, }, 0,0}, b_{-1,0}$ и $\tau_{1,0}$. Подчеркнем, что вычисление последних величин окончательно определяет постоянные $R_{0}^{\mathrm{ex}, 0,0}, A_{1,0}^{\mathrm{ex}}$,

$$
b_{1,0}=k_{0}\left(2 \tau_{1,0} R_{0}^{\mathrm{in}, 0,0} g^{\mathrm{ex}}\left(k_{0}\right)-b_{-1,0} q_{\omega}\right)
$$

и функцию $v_{1,0}^{\mathrm{ex}}$, а функцию $v_{1,0}^{\text {in }}$ определяет с точностью до слагаемого $A_{1,0}^{\text {in }}$, подчиненного уравнению (2.17). Более того, приравнивая асимптотики функций $v_{1,0}^{\text {in }(\text { ех })}$ при $\rho \rightarrow \infty$ и $\xi_{2}>0$ к рядам $V_{1.0}^{\text {in }(\mathrm{ex})}$, для остальных степеней определяем $\Pi_{0,0}^{\mathrm{in}(\mathrm{ex})}$ (т.е. коэфффициенты при старших производных в многочленах $\left.R_{i}^{\mathrm{in}(\mathrm{ex}), 2 i, 0}\right)$. И наконец, полагая

$$
v_{1,1}^{\text {in }} \equiv \frac{2 k_{0}}{\pi} R_{0}^{\text {in }, 0,0} \tau_{1,0}
$$

a $v_{1,1}^{\mathrm{ex}}$ в соответствии с (2.14) и приравнивая “асимптотики" этих функций к "рядам" $V_{1,1}^{\text {in }(\text { ex })}$ и "многочленам" $W_{1,1}^{\text {in (ex) }}$ при $\xi_{2}>0$ и $\xi_{2}<0$ соответственно, добиваемся равенств (2.12) и (2.13) для $N=1$ и находим значение (2.14) для $\tau_{2,1}$ (попутно определяя $\left.b_{1,1}\right)$. На этом закончился первьй шаг индукции, который помимо равенств $(2.12)$, (2.13) для $N=1$ дал два уравнения (2.16) и (2.19), связывающее три (поканеизвестные) постоянные $R_{0}^{\text {in }, 1,0}, A_{1,0}^{\text {in }}$ и $\tau_{2,0}$.

На втором шаге по $\widetilde{V}_{2,0}^{\text {in }(\text { ex })}$ и $\widetilde{W}_{2,0}^{\text {in(ex) }}$ определяем решения краевой задачи $(2.8)$ :

$$
v_{2,0}^{\text {in }}=R_{0}^{\text {in }, 0,0} \psi(0) \psi_{x_{1}}(0) Y+A_{2,0}^{\text {in }}, \quad v_{2,0}^{\mathrm{ex}} \equiv A_{2,0}^{\mathrm{ex}},
$$


где $A_{2,0}^{\text {in(ex) }}$ - некоторые постоянные. Приравнивая асимптотики $v_{2,0}^{\text {in }(\text { ех })}$ на бесконечности при $\xi_{2}<0$ к $W_{2,0}^{\text {in(ex) }}$, получаем равенства

$$
\begin{aligned}
A_{2,0}^{\mathrm{in}}+R_{0}^{\mathrm{in}, 0,0} \psi(0) \psi_{x_{1}}(0) q^{\omega} & =b_{-1,0} h\left(\frac{1}{2} \tau_{2,0} \tau_{1,0}+\tau_{3,0}\right)+b_{2,0} \\
A_{2,0}^{\mathrm{ex}} & =(-1)^{m} b_{2,0} .
\end{aligned}
$$

Аналогично, приравнивая асимптотики функций $v_{2,0}^{\operatorname{in}(\text { ex })}$ на бесконечности при $\xi_{2}>0$ до членов $O(1)$ включительно к $V_{2,0}^{\text {in(ex)}}$, получаем равенства

$$
\begin{gathered}
\tau_{1,0} R_{0}^{\mathrm{in}, 1,0}+\tau_{2,0} R_{0}^{\mathrm{in}, 0,0}=0, \\
\tau_{1,0} R_{0}^{\mathrm{ex}, 1,0}+\tau_{2,0} R_{0}^{\mathrm{ex}, 0,0}=0, \\
-2 k_{0} g^{\mathrm{ex}}\left(k_{0}\right)\left(\tau_{1,0} R_{0}^{\mathrm{ex}, 1,0}+\tau_{2,0} R_{0}^{\mathrm{ex}, 0,0}\right)=A_{2,0}^{\mathrm{ex}}, \\
a_{2,0,0}^{\text {in }} \psi^{2}(0)-2 k_{0} g^{\mathrm{in}}\left(k_{0}\right)\left(\tau_{1,0} R_{0}^{\mathrm{in}, 1,0}+\tau_{2,0} R_{0}^{\mathrm{in}, 0,0}\right)=A_{2,0}^{\mathrm{in}} .
\end{gathered}
$$

Из (2.25) и (2.26) находим $A_{2,0}^{\mathrm{ex}}$, что в силу (2.23) определяет $b_{2,0}$. Далее, разрешая систему уравнений $(2.16),(2.19),(2.24)$ (с учетом равенства $(2.21))$, определяем $\tau_{2,0}$ в соответствии с (2.14), а также постоянные $R_{0}^{\text {in, } 1,0}$ и $A_{1,0}^{\text {in }}$. Так как коэффициенты $R_{0}^{\text {in, }, 1,0}$ и $\tau_{2,0}$ определены, то, во-первьх, из (2.26) находим $R_{0}^{\mathrm{ex}, 1,0}$, а, во-вторых, приравнивая асимптотики функций $v_{2,0}^{\text {in }(\text { еx })}$ на бесконечности при $\xi_{2}>0$ к $V_{2,0}^{\text {in(ex) }}$ (для оставшихся членов), определяем все коэффициенты рядов $\Pi_{1,0}^{\text {in(ex) }}$ (т.е. старшие коэффициенты дифференциальных полиномов $R_{i}^{\text {in }(\text { ex }), 1+i, 0}$ при $\left.i \geqslant 1\right)$. Уравнения $(2.22),(2.27)$ являются прямыми аналогами уравнений (2.16) и (2.19) для определения (на следующем шаге) постоянных $A_{2,0}^{\text {in }}, \tau_{3,0}$ и $a_{2,0,0}^{\text {in }}$.

Дальнейшее доказательство проводится по индукции. $\mathrm{K}(N, k)$-шагу, где $k$ - показатель степени $\ln \varepsilon$, определяются все $\Pi_{q-2, s}^{\operatorname{in}(\mathrm{ex})}, \widetilde{W}_{q, s}^{\operatorname{in}(\mathrm{ex})}, \widetilde{V}_{q, s}^{\operatorname{in}(\mathrm{ex})} a_{q-1, s, 0}^{\operatorname{in}(\mathrm{ex})}, \tau_{q-1, s}, v_{q-2, s}^{\operatorname{in}(\mathrm{ex})}$, $v_{q-1, s}^{\mathrm{ex}}$ для $q \leqslant N$ и $s \leqslant k$, а функции $v_{N-1, s}^{\text {in }}$ определяются с точностью до постоянных слагаемых $A_{N-1, s}^{\text {in }}$, для которых имеются уравнения связи (аналоги $(2.22)$ и $\left.(2.27)\right)$ :

$$
A_{N-1, s}^{\text {in }}+B_{N-1, s}^{\text {in }}=b_{-1,0} h \tau_{N, s}, \quad a_{N-1, s, 0}^{\text {in }} \psi^{2}(0)+C_{N-1, s}^{\text {in }}=A_{N-1, s}^{\text {in }}, \quad s \leqslant N-1,
$$

где $B_{N-1, s}^{\text {in }}$ и $C_{N-1, s}^{\text {in }}$ - вполне определенные числа. На $(N, s)$-м шаге при $s<N$ по $\widetilde{W}_{N, s}^{\mathrm{in}(\mathrm{ex})}$ и $\widetilde{V}_{N, s}^{\mathrm{in}(\mathrm{ex})}$ определяем $v_{N, s}^{\mathrm{in}(\mathrm{ex})}$ равенствами

$$
v_{N, s}^{\mathrm{in}(\mathrm{ex})}=\widetilde{v}_{N, s}^{\mathrm{in}(\mathrm{ex})}+A_{N, s}^{\mathrm{in}(\mathrm{ex})}
$$

где $A_{N, s}^{\text {in(ex) }}$ - пока произвольные постоянные, a $\widetilde{v}_{N, s}^{\operatorname{in}(\text { ex })}-$ решения краевых задач $(2.8)$, имеющих асимптотики

$$
\begin{aligned}
& \widetilde{v}_{N, s}^{\mathrm{in}(\mathrm{ex})}=\widetilde{W}_{N, s}^{\mathrm{in}(\mathrm{ex})}+D_{N, s}^{\mathrm{in}(\mathrm{ex})}+o(1), \quad \xi_{2}<0, \\
& \widetilde{v}_{N, s}^{\mathrm{in}(\mathrm{ex})}=\widetilde{V}_{N, s}^{\mathrm{in}(\mathrm{ex})}+E_{N, s}^{\mathrm{in}(\mathrm{ex})} \ln \rho+F_{N, s}^{\mathrm{in}(\mathrm{ex})}+O\left(\rho^{-1}\right), \quad \xi_{2}>0,
\end{aligned}
$$


где $D_{N, s}^{\text {in }(\text { ex })}, E_{N, s}^{\text {in }(\text { ex })}$ и $F_{N, s}^{\text {in(ex) }}$ - также некоторые вполне определенные постоянные. Существование таких функций следует из утверждений лемм 2.1 и 2.2. Приравнивая асимптотики $v_{N, s}^{\text {in }(\mathrm{ex})}$ при $\rho \rightarrow \infty$ и $\xi<0$ к многочленам $W_{N, s}^{\text {in }(\mathrm{ex})}$, получаем пару уравнений

$$
\begin{aligned}
& A_{N, s}^{\mathrm{in}}+D_{N, s}^{\mathrm{in}}=b_{-1,0} h\left(\frac{1}{2} \tau_{N, s} \tau_{1,0}+\tau_{N+1, s}\right)+b_{N, s}, \\
& A_{N, s}^{\mathrm{ex}}+D_{N, s}^{\mathrm{ex}}=(-1)^{m} b_{N, s} .
\end{aligned}
$$

Аналогично, приравнивая асимптотики функций $v_{N, s}^{\text {in(ex) }}$ на бесконечности при $\xi_{2}>0$ до членов $O(1)$ включительно к $V_{N, s}^{\text {in }(\mathrm{ex})}$, получаем равенства

$$
\begin{gathered}
\frac{2 k_{0}}{\pi}\left(\tau_{1,0} a_{N-1, s, 0}^{\mathrm{in}}+\tau_{N, s} R_{0}^{\mathrm{in}, 0,0}\right)=E_{N, s}^{\mathrm{in}}, \\
\frac{2 k_{0}}{\pi}\left(\tau_{1,0} a_{N-1, s, 0}^{\mathrm{ex}}+\tau_{N, s} R_{0}^{\mathrm{ex}, 0,0}\right)=E_{N, s}^{\mathrm{ex}}, \\
-2 k_{0} g^{\mathrm{ex}}\left(k_{0}\right)\left(\tau_{1,0} a_{N-1, s, 0}^{\mathrm{ex}}+\tau_{N, s} R_{0}^{\mathrm{ex}, 0,0}\right)=A_{N, s}^{\mathrm{ex}}, \\
a_{N, s, 0}^{\mathrm{in}} \psi^{2}(0)-2 k_{0} g^{\mathrm{in}}\left(k_{0}\right)\left(\tau_{1,0} a_{N-1, s, 0}^{\mathrm{in}}+\tau_{N, s} R_{0}^{\mathrm{in}, 0,0}\right)=A_{N, s}^{\mathrm{in}} .
\end{gathered}
$$

Из (2.32) и (2.33) находим $A_{N, s}^{\mathrm{ex}}$, что в силу (2.30) определяет $b_{N, s}$. Далее, разрешая систему уравнений $(2.28),(2.32)$, определяем $\tau_{N, s}, a_{N-1, s, 0}^{\text {in }} A_{N-1, s}^{\text {in }}$. Так как коэффициенты $a_{N-1, s, 0}^{\text {in }} \tau_{N, s}$ определены, то, во-первых, из $(2.33)$ находим $a_{N, s, 0}^{\text {ex }}$, a, во-вторых, приравнивая асимптотики функций $v_{N, s}^{\text {in }(\text { ex })}$ на бесконечности при $\xi_{2}>0$ к $V_{N, s}^{\text {in(ex) }}$ (для оставшихся членов), определяем все коэффициенты рядов $\Pi_{N-1, s}^{\text {in(ex) }}$. Уравнения (2.29), $(2.34)$ являются "заменой” $(2.28)$ для $(N+1, s)$-го шага.

Эта же процедура повторяется на $(N, s+1)$-м шаге (если $s+1<N)$. На $(N, N)$-м шаге ситуация еще проще. Непосредственно из леммы 2.1 следует, что

$$
\begin{aligned}
v_{N, N}^{\mathrm{in}} & \equiv \frac{2 k_{0}}{\pi} \sum_{t=0}^{N-1} R_{0}^{\mathrm{in}, t, t} \tau_{k-t, k-t-1}=\tau_{N+1, N} b_{-1,0} h+b_{N, N} \\
v_{N, N}^{\mathrm{ex}} & \equiv \frac{2 k_{0}}{\pi} \sum_{t=0}^{N-1} R_{0}^{\mathrm{ex}, t, t} \tau_{k-t, k-t-1}=(-1)^{m} b_{N, N} .
\end{aligned}
$$

Разрешая (2.35), находим $b_{N, N}$ и $\tau_{N+1, N}$. Теорема доказана.

Подчеркнем, что в теореме построены две серии асимптотических рядов, соответствующие $\tau_{1,0}=(-1)^{n} \psi(0)(|\omega| / 2 h)^{1 / 2}, n=1,2$ (или, что то же самое, $R_{0}^{\text {in }, 0,0}=$ $(-1)^{n} \psi_{0}^{-1} 2^{-1 / 2}$. Кроме этого, из формул (2.14) следуют (пока на формальном уровне) формулы (1.8) для $\tau_{1,0}$ и $\tau_{2,1}$ и равенство

$$
\tau_{2,0}^{(n)}=\frac{1}{2} \frac{|\omega|}{h}\left(\frac{2 k_{0}}{\pi}\left(\ln \left(\frac{2|\omega|}{\pi}\right)-1\right)-\frac{1}{4} \psi^{2}(0)-k_{0}\left(g^{\mathrm{in}}\left(k_{0}\right)+g^{\mathrm{ex}}\left(k_{0}\right)\right)\right) .
$$

Из последнего равенства, учитывая, что $\operatorname{Im} g^{\text {in }}\left(k_{0}\right)=0, \operatorname{Im} g^{\text {ex }}\left(k_{0}\right)=k_{0} \sigma$ (последнее см., например, в [14], [16]), получаем и формулу (1.8) для $\operatorname{Im} \tau_{2,0}$. И, наконец, из (2.14) 
следует, что у рядов (2.1)-(2.3) главные члены имеют вид, указанный для функций $\Psi_{\varepsilon}^{(n)}$ в пункте в) теоремы 1.1 в соответствующих областях.

Обозначим

$$
\begin{aligned}
\psi_{\varepsilon, N}^{(n)}(x, k)= & \chi\left(\frac{\left|x^{\mathrm{in}}\right|}{\varepsilon^{1 / 2}}\right) \sum_{i=0}^{N} \sum_{j=0}^{i} \varepsilon^{i / 2} \ln ^{j} \varepsilon\left(k_{0}^{2}-k^{2}\right) R_{[(i-j) / 2]}^{\mathrm{in}, i, j}\left(D_{y}\right) G^{\mathrm{in}}\left(x, x_{0}^{\mathrm{in}}, k\right) \\
& +\chi\left(\frac{\left|x^{\mathrm{ex}}\right|}{\varepsilon^{1 / 2}}\right) \sum_{i=0}^{N} \sum_{j=0}^{i} \varepsilon^{i / 2} \ln ^{j} \varepsilon\left(k_{0}^{2}-k^{2}\right) R_{[(i-j) / 2]}^{\mathrm{ex}, i, j}\left(D_{y}\right) G^{\mathrm{ex}}\left(x, x_{0}^{\mathrm{ex}}, k\right) \\
& +\left(1-\chi\left(\frac{\mid x^{\mathrm{in}}}{\mid \varepsilon^{1 / 2}}\right)\right) v_{\varepsilon, N / 2}^{\mathrm{in}}\left(\frac{x^{\mathrm{in}}}{\varepsilon}\right)+\left(1-\chi\left(\frac{\mid x^{\mathrm{ex}}}{\mid \varepsilon^{1 / 2}}\right)\right) v_{\varepsilon, N / 2}^{\mathrm{ex}}\left(\frac{x^{\mathrm{ex}}}{\varepsilon}\right) \\
& +\chi\left(\frac{\left|x^{\mathrm{in}}\right|}{\varepsilon^{1 / 2}}\right) \chi\left(\frac{\left|x^{\mathrm{ex}}\right|}{\varepsilon^{1 / 2}}\right) w_{\varepsilon, N / 2}(x),
\end{aligned}
$$

где $w_{M, \varepsilon}-$ частичная сумма ряда $(2.2), \chi(t)$ - гладкая срезающая функция, равная нулю при $t<0$ и единице при $t>2$, а индекс $n=1,2$ в левой части соответствует двум сериям асимптотик и для краткости опущен в правой части. Из теоремы 2.1 стандартным образом (см., например, [17]) вытекает

СлЕДСТВИЕ. Пусть асимптотики функиий $\tau_{\varepsilon}^{(n)}$ и ряды (2.1)-(2.3) удовлетворяют утверждениям теоремы. Тогда

(a) $\psi_{\varepsilon, N}^{(n)}(x, k) \in C^{\infty}\left(\Omega_{\varepsilon}\right)$ есть голоморфная функиия из $W_{2, \mathrm{loc}}^{1}\left(\Omega_{\varepsilon}\right), n p u \operatorname{Im} k \geqslant 0$ удовлетворяюияая (1.2);

(б) $\psi_{\varepsilon, N}^{(n)}(x, k)$ является решением $(1.1)$, әде $F(x, k)=F_{\varepsilon, N}^{(n)}(x, k)$ - голоморфная функиия из $L_{2}\left(\mathbb{R}^{2}\right), \operatorname{supp} F_{\varepsilon, N} \subset \varkappa_{\varepsilon} \cup S^{\operatorname{in}}\left(2 \varepsilon^{1 / 2}\right) \cup S^{\operatorname{ex}}\left(2 \varepsilon^{1 / 2}\right) u$

$$
\left\|F_{\varepsilon, N}^{(n)}\left(\circ, \tau_{\varepsilon}^{(n)}\right)\right\|_{L_{2}\left(\mathbb{R}^{2}\right)} \leqslant C_{N} \varepsilon^{N_{1}}
$$

где $N_{1}$ растет неограниченно вместе с $N$.

Из явного вида главных членов асимптотик (2.14) вытекает также, что

$$
\left\|\psi_{\varepsilon, N}^{(n)}\left(\cdot, \tau_{\varepsilon}^{(n)}\right)\right\|_{L_{2}\left(\Omega_{\varepsilon} \cap S(T)\right)} \rightarrow 1, \quad \int_{\Omega_{\varepsilon} \cap S(T)} \psi_{\varepsilon, N}^{(1)}\left(x, \tau_{\varepsilon}^{(1)}\right) \psi_{\varepsilon, N}^{(2)}\left(x, \tau_{\varepsilon}^{(2)}\right) d x \rightarrow 0
$$

при $\varepsilon \rightarrow 0$ для любого достаточно большого $T$.

Построение формальных асимптотик закончено.

3. Обоснование асимптотик. Из [3] следует

Лемма 3.1. Cуществует

(а) не более двух полюсов $\tau_{\varepsilon}^{(n)}$, сходящихся при $\varepsilon \rightarrow 0 \kappa k_{0} \in \Sigma_{1}^{\text {in }} \cap \Sigma^{\mathrm{ch}}$;

(б) если $\tau_{\varepsilon}^{(1)} \neq \tau_{\varepsilon}^{(2)}$, то каждому из них соответствует одна обобщенная собственная функиия.

В свою очередь, следуя [1], [16] и используя лемму 3.1, нетрудно показать справедливость следуюшего утверждения. 
Лемма 3.2. Пусть $k_{0} \in \Sigma_{1}^{\text {in }} \cap \Sigma^{\mathrm{ch}}, F \in L_{2}\left(\mathbb{R}^{2}\right) u \operatorname{supp} F \subset S(R)$. Тогда

(а) для аналитического продолхения решения краевой задачи справедлива равномерная по малым $\varepsilon$ и $k$, близким $\kappa k_{0}$, оценка

$$
\left\|u_{\varepsilon}\right\|_{L_{2}(S(T))} \leqslant \frac{C(R, T)}{\left|\left(\tau_{\varepsilon}^{(1)}-k\right)\left(\tau_{\varepsilon}^{(2)}-k\right)\right|}\|F\|_{L_{2}\left(\mathbb{R}^{2}\right)},
$$

не зависящая от совпадения или несовпадения полюсов $\tau_{\varepsilon}^{(n)}$;

(б) если $\tau_{\varepsilon}^{(1)} \neq \tau_{\varepsilon}^{(2)}$, то справедливо утверждение пункта б) теоремы 1.1 и имеет место равномерная по малым $\varepsilon и k \in K$, близким $\kappa k_{0}$, оценка

$$
\left\|\widetilde{u}_{\varepsilon}\right\|_{L_{2}(S(T))} \leqslant C(R, T)\|F\|_{L_{2}\left(\mathbb{R}^{2}\right)}
$$

(в) $е с л и \tau_{\varepsilon}^{(1)} \neq \tau_{\varepsilon}^{(2)}$ и для любого достаточно больиого $T>0$

$$
\begin{aligned}
& \frac{1}{\left\|\Psi_{\varepsilon}^{(1)}\right\|_{L_{2}\left(\Omega_{\varepsilon} \cap S(T)\right)}\left\|\Psi_{\varepsilon}^{(2)}\right\|_{L_{2}\left(\Omega_{\varepsilon} \cap S(T)\right)}} \int_{\Omega_{\varepsilon} \cap S(T)} \Psi_{\varepsilon}^{(1)} \Psi_{\varepsilon}^{(2)} d x \rightarrow 0, \quad \varepsilon \rightarrow 0, \\
& m o\left\|\Psi_{\varepsilon}^{(n)}\right\|_{L_{2}\left(\Omega_{\varepsilon} \cap S(R)\right)} \rightarrow 1 n p u \varepsilon \rightarrow 0 .
\end{aligned}
$$

ДОКАЗАТЕЛЬСТво ТЕОРЕмЫ 1.1. Справедливость пункта а) следует из пункта (а) леммы 3.2, следствия теоремы 2.1 и произвола в выборе $N$. Так как по построению $\tau_{\varepsilon}^{(1)} \neq \tau_{\varepsilon}^{(2)}$, то из пункта (б) леммы 3.2 вытекает справедливость пункта б) доказываемой теоремы. Далее, из пункта (б) леммы 3.2, следствия теоремы 2.1 и произвола в выборе $N$ следует, что в представлении (1.9)

$$
\Psi_{\varepsilon}^{(n)}(x)=\alpha_{n}(\varepsilon) \psi_{\varepsilon}^{(n)}(x)
$$

где $\psi_{\varepsilon}^{(n)}$ имеет асимптотические разложения (2.1)-(2.3), коэффициенты которых удовлетворяют утверждениям теоремы 2.1, а $\alpha_{n}(\varepsilon)$ - некоторый нормирующий множитель. В свою очередь, из (2.36) и пункта (в) леммы 3.2 следует, что

$$
\alpha_{n}(\varepsilon)=1+o(1), \quad \varepsilon \rightarrow 0
$$

В силу $(3.1),(3.2)$ и (2.14) получаем справедливость пункта в) теоремы 1.1. 


\section{СПИСОК ЦИТИРОВАННОЙ ЛИТЕРАТУРЫ}

[1] Beale J.T. Scattering frequencies of resonator // Comm. Pure Appl. Math. 1973. V. 26. P. 549-564.

[2] Арсеньев А. А. Об особенностях аналитического продолжения и резонансных свойствах решения задачи рассеяния для уравнения Гельмгольца // ЖВМиМФ. 1971. Т. 12. С. 112-138.

[3] Brown R. M., Hislop P. D., Martinez A. Eigenvalues and resonances for domains with tubes: Neumann boundary conditions // J. Diff. Equations. 1995. V. 115. P. 458-476.

[4] Гадыльшин Р.P. О рассеянии на цилиндре с узкой щелью и стенками конечной толщины // ТМФ. 1996. Т. 106. №1. С. 24-43.

[5] Ван-Дайк М. Методы возмущений в механике жидкости. М.: Мир, 1967.

[6] Найфе А. Х. Методы возмущений. М.: Мир, 1986.

[7] Ильин А. М. Согласование асимптотических разложений решений краевых задач. М.: Наука, 1989.

[8] Gadyl'shin R. R. On the scattering of $H$-polarized electromagnetic field by an ideally conductive cylindrical body of a trapping type // C. R. Acad. Sci. Paris Sér. IIb. 2001. V. 329. P. 137-140.

[9] Санчес-Паленсиа Е. Неоднородные среды и теория колебаний. М.: Мир, 1985.

[10] Lord Rayleigh. The theory of Helmholtz resonator // Proc. Royal Soc. London. 1916. V. 92. P. 265-275.

[11] Арсеньев А. А. О существовании резонансных полюсов и резонансов при рассеянии в случае краевых условий II и III рода // ЖВМиМФ. 1976. Т. 16. № 3. С. 718-724.

[12] Gadyl'shin R. R. On scattering frequencies of acoustic resonator // C. R. Acad. Sci. Paris Sér. I. 1993. V. 316. P. 959-963.

[13] Gadyl'shin R. R. Asymptotics of scattering frequencies with small imaginary parts for acoustic resonator // Math. Modelling Numer. Anal. 1994. V. 28. № 6. P. 761-780.

[14] Гадыльшин Р. Р. Двумерньй аналог резонатора Гельмгольца с идеально жесткими стенками // Дифференц. уравнения. 1994. Т. 30. С. 221-229.

[15] Лаврентьев М.А., Шабат Б. В. Методы теории функций комплексного переменного. М.: Наука, 1973.

[16] Гадыльшин Р. Р. О полюсах акустического резонатора // Функцион. анализ и его прилож. 1993. № 5. C. 3-16.

[17] Gadyl'shin R.R. On acoustic Helmholtz resonator and on its electromagnetic analogue // J. Math. Phys. 1994. V. 35. P. 3464-3481.

Башкирский государственный педагогический университет, г. Уфа

Поступило

E-mail : gadylshin@bspu.ru

06.04.2001 